\title{
Alternation of different fluctuation regimes in the stock market dynamics
}

\author{
J. Kwapień ${ }^{1,2}$, S. Drożdż $\dot{1}^{1-3}$ and J. Speth ${ }^{1}$ \\ ${ }^{1}$ Institut für Kernphysik, Forschungszentrum Jülich, D-52425 Jülich, Germany \\ ${ }^{2}$ Institute of Nuclear Physics, PL-31-342 Kraków, Poland \\ ${ }^{3}$ Institute of Physics, University of Rzeszów, PL-35-310 Rzeszów, Poland
}

\begin{abstract}
Based on the tick-by-tick stock prices from the German and American stock markets, we study the statistical properties of the distribution of the individual stocks and the index returns in highly collective and noisy intervals of trading, separately. We show that periods characterized by the strong inter-stock couplings can be associated with the distributions of index fluctuations which reveal more pronounced tails than in the case of weaker couplings in the market. During periods of strong correlations in the German market these distributions can even reveal an apparent Lévy-stable component.
\end{abstract}

Key words: Financial market, Central Limit Theorem, Correlation matrix, Stylized facts

PACS: 89.20.-a, 89.65.Gh, 89.75.-k

\section{Introduction}

A series of papers devoted to the analysis of financial data fluctuations disclosed that the corresponding distributions can be characterized by the Paretian scaling $[1,2,3,4,5]$. These studies, based on the large data sets of historical stock prices and on the index values, showed that both the distributions of stock price fluctuations and the distriutions of index returns reveal scaling over a broad range of time scales from minutes to days (although a more recent investigation found that scaling is restricted to rather short time scales [6]). A remarkable related issue is that the stocks and indices exhibit similar value of the scaling exponent $\alpha \simeq 3.0[4,5]$. In accordance with the Central Limit Theorem, the distribution of a random variable being a sum of a number of iid random variables with a finite second moment, has to converge to a normal distribution. From this point of view, the similarity of the distributions for the 
stocks and the corresponding indices requires that the financial data violate the assumptions of the theorem. And as these data have indeed finite variance, a plausible cause for the problems with the convergence can be related to the correlations among the data. This claim seems to be supported by findings that an artificial S\&P index constructed from randomly reshuffled stock returns, presents a much better convergence to a Gaussian than the original index $[4]$.

An appropriate measure of correlations among elements of a system is the spectrum of the correlation matrix eigenvalues, which can be easily compared with the universal properties of random matrices [7]. A few recent works have shown that the financial market can be described by at least one repelled eigenvalue with a magnitude exceeding the likely range of values allowed for a random matrix. This one or more deviating eigenvalues indicate that there are relations between various components of the market $[8,9,10,11]$.

The main purpose of the present work is a quantitative description of the possible relation between the stock price movements and the properties of the distribution of the corresponding index fluctuations. We showed in a previous analyses which were focused on daily patterns of the German DAX index fluctuations that certain characteristic time intervals of a trading day with high index volatility are associated with fluctuation distributions with properties different from more silent intervals of trading $[12,13]$. Since high volatility is connected with stronger correlations between the stocks $([14,15])$ we expect that strong and weak inter-stock correlations are reflected in different properties of the index fluctuations. By choosing a few distinct time scales (1-30 minutes) we are able to test the stability of the results.

\section{Methodology}

Our analysis is based on the high frequency tick-by-tick data covering the two years 1998-99 period and comprising the recordings of 30 companies included in the Dow Jones Industrial Average and 30 companies included in the German DAX30 index, together with the two indices [16]. Inevitably, such a long interval of time comprises some changes of the index composition. We decided that only those stocks which were a part of an index for the majority of time, can be taken into consideration. Along this way, for the whole interval under study we analyze the data for the individual companies CHV, GT, S and UK, although on Nov 1, 1999 they were replaced in DJIA by HD, INTC, MSFT and SBC. In a similar manner for the German market, we analyze ADS instead of BVM (delisted due to its fussion with BHW). For the German stock market we have roughly $30 \%$ more data points, because of a longer trading day in Frankfurt (8:30 hours vs. 6:30 hours in New York). The data has been 
preliminary processed to clear out recording errors.

Let us assume we have a set of $N$ assets and $x_{\beta}\left(t_{i}\right)(i=1, \ldots, T)$ is a price of the $\beta$-th asset at instant $t_{i}$. The corresponding time series of normalized logarhitmic price returns reads:

$$
g_{\beta}\left(t_{i}\right)=\frac{G_{\beta}\left(t_{i}\right)-\left\langle G_{\beta}\left(t_{i}\right)\right\rangle_{t_{i}}}{\sigma\left(G_{\beta}\right)}, \quad \sigma\left(G_{\beta}\right)=\sqrt{\left\langle G_{\beta}^{2}\left(t_{i}\right)\right\rangle_{t_{i}}-\left\langle G_{\beta}\left(t_{i}\right)\right\rangle_{t_{i}}^{2}}
$$

where

$$
G_{\beta}\left(t_{i}\right)=\ln x_{\beta}\left(t_{i}+\Delta t\right)-\ln x_{\beta}\left(t_{i}\right)
$$

The time lag $\Delta t$ defines a time scale and $\langle\ldots\rangle_{t_{i}}$ stands for averaging over discrete time. From all the time series $g_{\beta}\left(t_{i}\right)(\beta=1, \ldots, N ; i=1, \ldots, T)$ we construct an $N \times T$ data matrix $\mathbf{M}$ and then calculate a correlation matrix $\mathbf{C}$ defined by

$$
\mathbf{C}=(1 / T) \mathbf{M M}^{\mathrm{T}}
$$

which is is an $N \times N$ square matrix with correlation coefficients as its entries.

After the correlation matrix is calculated, we diagonalize it and obtain a spectrum of its eigenvalues $\lambda_{k}(k=1, \ldots, N)$. For a random matrix (the so-called Wishart matrix) constructed from series of random numbers taken from the normal distribution, in the limit of $N \rightarrow \infty$ there exist exactly $N$ non-zero eigenvalues, providing $Q:=T / N>1$ (and $N-1$ ones for $Q=1$ ). In this case an analytic expression for the distribution of the matrix eigenvalues exists [17]:

$$
\begin{aligned}
& \rho_{C}(\lambda)= \frac{Q}{2 \pi \sigma^{2}} \frac{\sqrt{\left(\lambda_{\max }-\lambda\right)\left(\lambda-\lambda_{\min }\right)}}{\lambda}, \\
& \lambda_{\min }^{\max }=\sigma^{2}(1+1 / Q \pm 2 \sqrt{1 / Q})
\end{aligned}
$$

with $\lambda_{\min } \leq \lambda \leq \lambda_{\max }$, and where $\sigma^{2}$ is equal to the variance of the time series. In both cases, any deviation from the universal Random Matrix Theory predictions means that the correlation matrix comprises some genuine information specific for the system under study.

In a system like the stock market, the correlation matrix usually reveals at least one strongly repelled eigenvalue, describing the common behaviour of a group of assets or even the common evolution of the whole market $[8,9,10,18,19]$. The magnitude of such an eigenvalue is related to the range of correlation of different asset prices. A more collective market behaviour is reflected in 
a larger $\lambda_{1}$. In the real markets the range of the inter-stock couplings turns out to be strongly time-dependent; this refers both to long time scales (daily returns) [10,20] and to extremely short ones (of order of minutes and even seconds) [12,13]. As it has already been mentioned, the correlations grow in highly volatile periods of time and fade during more silent intervals, when trading is dominated by noise (see [10], but also [20]). The sudden elevation of the largest eigenvalue in crash times and decrease in onset periods of a rally can serve as examples of such behaviour.

A possible influence of the asset coupling strength on the distribution of index returns may be observed by comparing returns corresponding to intervals with strong correlations (large values of $\lambda_{1}$ ) and those from intervals with weak correlations (small $\lambda_{1}$ ). To accomplish this, we divide the whole two-years-long period under study into equal disjoint time windows $w_{j}, j=1, \ldots, n_{w}$ each of length $T_{w}$. In each window we calculate the correlation matrix $\mathbf{C}$ and its largest eigenvalue $\lambda_{1}\left(w_{j}\right)$. Next we determine the eigenvalue distribution $P\left(\lambda_{1}\left(w_{j}\right)\right)$ and select such windows $w_{k}$ that $\lambda_{1}\left(w_{k}\right)$ falls within a specific range of values of the $P\left(\lambda_{1}\left(w_{j}\right)\right)$ distribuant. Finally, we compute the distributions of index and stock returns belonging to these selected windows. Since we need a good time resolution for fixed $N=30$, we choose $T_{w}=30$ (i.e. $Q=T_{w} / N=1$ ), regardless of the time scale $\Delta t$. From the RMT perspective this fixes the upper edge of the random eigenvalues bulk at exactly $\lambda_{\max }=4.0$. However, since we use very short windows $(N \ll \infty)$, the average value of $\lambda_{1}$ may be smaller than $\lambda_{\text {max }}$ in the random case and $\lambda_{1}\left(w_{j}\right)$ may fluctuate around the average for different $j$. (Fortunately, these fluctuations described by the so-called TracyWidom distribution $[21,22]$ are relatively small already for $N=30$ and thus do not influence our findings for $\lambda_{1} \gg \lambda_{\max }$.) For our analysis, four distinct time scales were chosen: $\Delta t=1, \Delta t=5, \Delta t=10$ and $\Delta t=30$ minutes; we could not include any higher scales due to extremely poor statistics of returns in that case.

\section{Results}

The upper panels of Figure 1 present the time course of the DAX (a) and of the DJIA (b) indices (here sampled every 10 minutes) in the studied time interval, i.e. between Dec 1, 1997 and Dec 31, 1999. Over this period, both indices experienced a significant increase: $\simeq 70 \%$ in the case of DAX and $\simeq$ $40 \%$ in the case of DJIA, although large drowdowns were also observed (e.g. in August 1998). In principle, however, that was exactly this period in which the most powerful bull market was observed both at NYSE and at Deutsche Börse. For a comparison, the lower panels display the largest eigenvalue for each time window $\lambda_{1}\left(w_{j}\right)$ as a function of time; each point corresponds to a single time window $w_{j}$ which is $300 \mathrm{~min}$ (30 data points) long in the present 
(a)

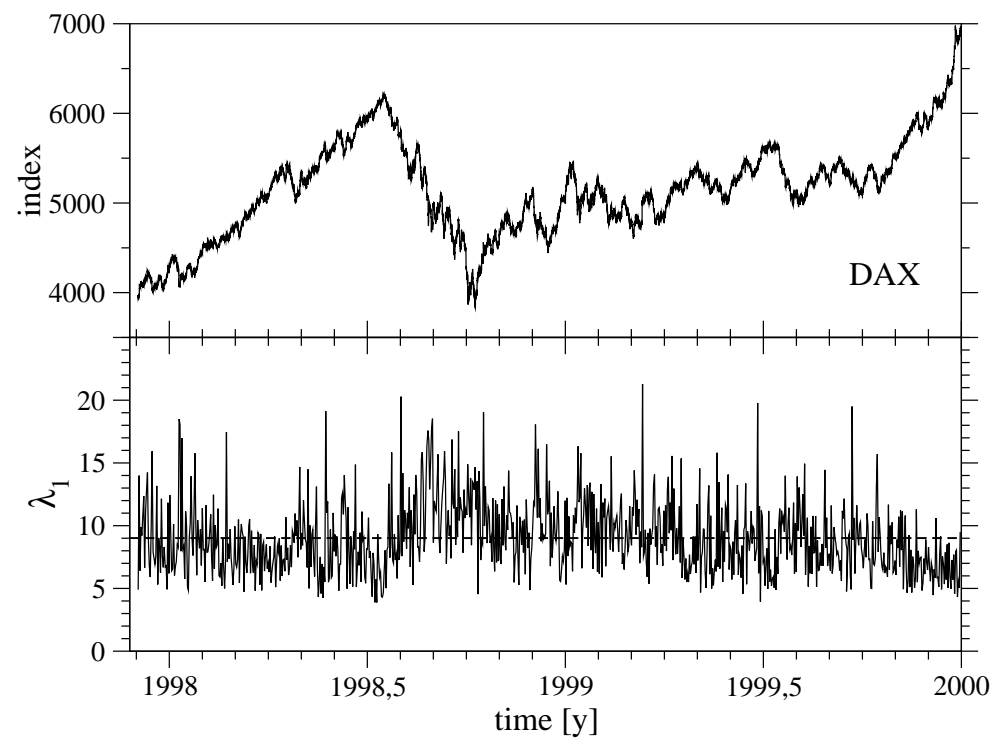

(b)

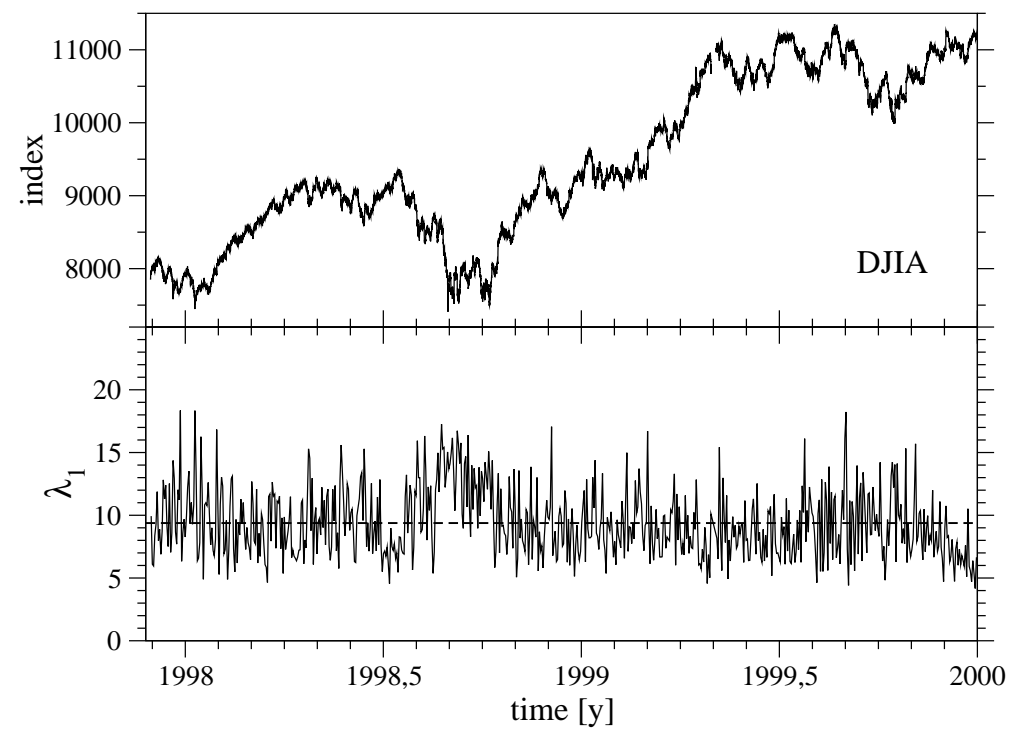

Fig. 1. Time course of the DAX index (a) and of the DJI Average (b) sampled with 10 min frequency (upper panels) together with the corresponding $\lambda_{1}\left(w_{j}\right)$-values calculated in time windows of $T_{w}=30$ data points (lower panels). The arithmetic average of the eigenvalue over all the windows is also indicated (dashed line).

case. The varying degree of collectivity is clearly visible here. This resembles the evolution of $\lambda_{1}$ calculated from daily data in ref. [10]. For the sake of clarity of the Figure, we chose $\Delta t=10 \mathrm{~min}$, since smaller time scales are associated with more noisy dynamics. Comparing the lower panels of (a) and (b), we see that both the markets have similar average value of $\lambda_{1}$ (denoted by horizontal lines) equal to 9.01 (DAX) and 9.38 (DJIA), respectively. This, 
however, cannot be treated as a rule, because for shorter $\Delta t \mathrm{DAX}$ is on average significantly more collective than DJIA. Even for this time scale of 10 min the German market develops $\lambda_{1}$ which reaches extremely high values significantly more often than it happens in the Dow Jones market (compare ref. [10]). The difference between the values of $\lambda_{1}$ in the strongly collective and the least collective periods of time is striking. The smallest $\lambda_{1}\left(w_{j}\right)$ 's fall inside the noisy part of the eigenvalue spectrum $\left(\lambda_{1} \simeq \lambda_{\max }\right)$ suggesting that no meaningful correlations are present at that time, while, on the other hand, the largest $\lambda_{1}\left(w_{j}\right)$ 's, almost saturating the available range of values $\left(\lambda_{1} \leq \operatorname{Tr} \mathbf{C}=N\right)$, describe nearly "rigid" market.

In order to compare the statistical properties of the stock and of the index returns across windows with different degree of inter-stock couplings, we introduce a pair of parameters $\zeta^{W}$ and $\zeta^{S}$ defined by the following relation

$$
\zeta^{W, S}:=\frac{\#\left\{w_{j}: \lambda_{1}\left(w_{j}\right)<\Lambda^{W, S}\right\}}{n_{w}}, \quad\left(\zeta^{W} \leq \zeta^{S}\right)
$$

where $\Lambda^{S}$ denotes the lower threshold for $\lambda_{1}$, defining the strongly correlated market and $\Lambda^{W}$ denotes the upper threshold when the market is considerably weakly correlated. Specific values of $\Lambda^{W}, \Lambda^{S}$ depend on a particular choice of $\zeta^{W}, \zeta^{S}$. By this definition, the case of $\zeta^{W}=\zeta^{S}=0.5$ corresponds to the median of the distribution, while the one of $\zeta^{W}=0.2, \zeta^{S}=0.8$ to the 20 th and 80th percentile of the distribution, respectively. From now on, we will recognize two cases: periods of collective trading (strong stock cross-correlations, $S$ ) if $\lambda_{1}\left(w_{j}\right)>\Lambda^{S}$ and periods of uncorrelated trading (weak cross-correlations, $W$ ) if $\lambda_{1}\left(w_{j}\right)<\Lambda^{W}$.

We anticipate that each of these two cases is represented by a distribution of index returns with somewhat different properties. An index can be either a simple sum of the related stock prices (e.g. Dow Jones) or a sum of prices weighted by capitalization of the corresponding companies (e.g. DAX and S\&P indices family). This suggests that the index fluctuations may be described by a distribution being closer to a Gaussian in periods of uncorrelated trading, when a lack of profound correlations brings situation close to the assumptions of the Central Limit Theorem, while being significantly different from normal during strongly collective stock behaviour, when these assumptions are firmly violated. In contrast, the properties of the corresponding distributions for the individual stock returns may not be so sensitive to the inter-stock correlations as the index returns are.

Before we divide the signals into the correlated and noisy parts, we show in Figure 2 the cumulative distributions of the normalized stock returns and of the index returns for the DAX market (Fig. 2(a)) and the DJI market (Fig. 2(b)). This Figure allows one to compare the distribution of the stock returns calcu- 

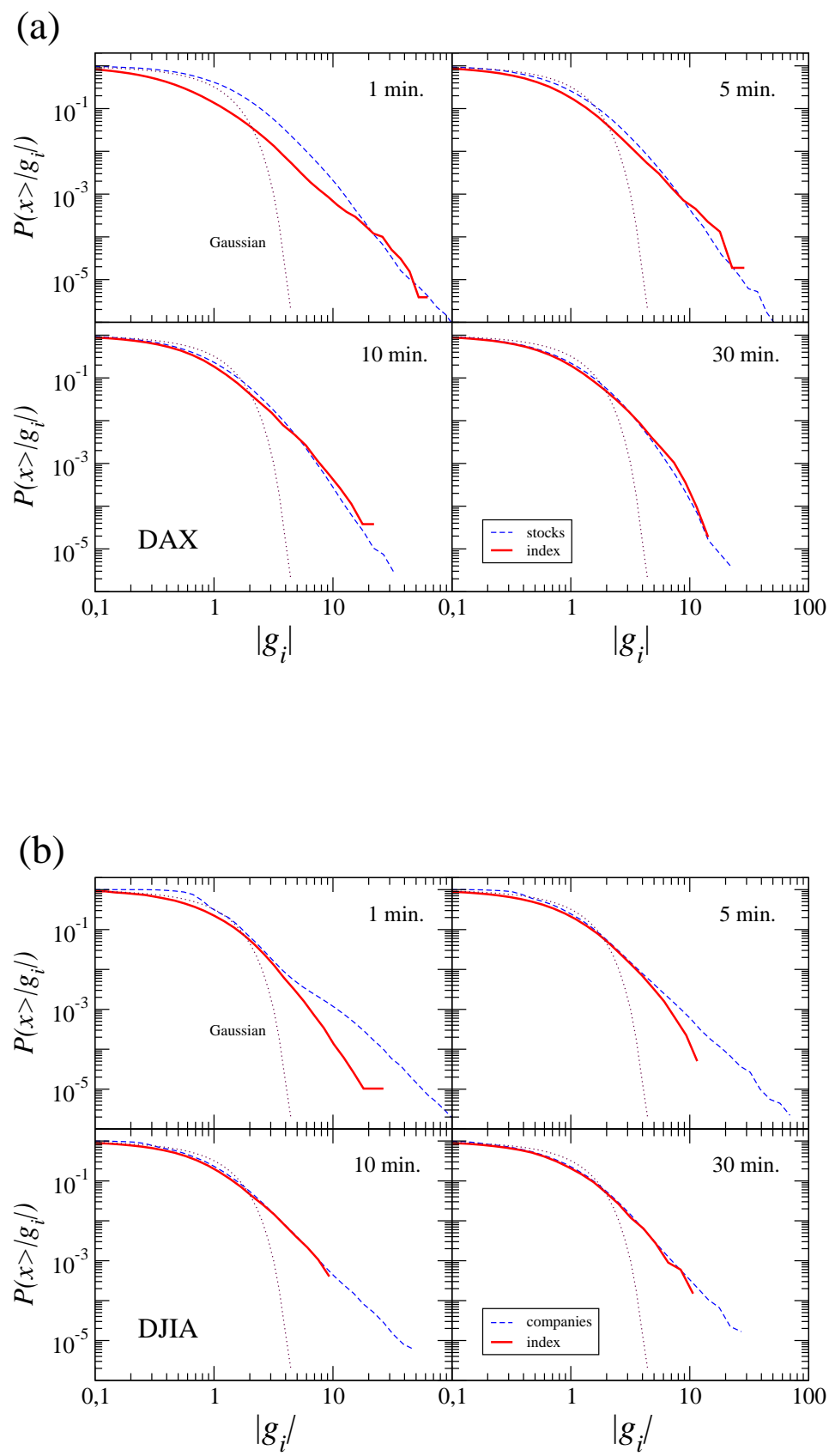

Fig. 2. Cumulative distributions of normalized stock returns (dashed) and of normalized index returns (solid) for four different time scales (1-30 min) and the two markets studied: DAX (a) and DJIA (b). The cumulative normal distribution is indicated by a dotted line in each panel.

lated for all 30 companies constituting each of the markets (dashed line) with the distribution of the index returns (solid line), for four different $\Delta t$. As it can be seen in Figure, the distributions of the stock returns scale in their tails for both the markets and for all the time scales. For the two shortest time scales the tails of the distributions of the index returns for DAX and for DJIA 
have different properties, however. On one hand, in DAX, the slope of the distribution of the index returns is smaller than the slope of the corresponding distribution of the stock returns. On the other hand, the reverse effect is observed for DJI stocks and DJIA. Interestingly, by increasing $\Delta t$ from 1 to 30 min the difference between the index returns distribution and the associated stock returns distribution disappears for both DAX and DJIA. Nevertheless, in each case in Fig. 2 all the distributions are far from Gaussian.

Later on, we shall discuss the origin of the difference in the slope of the tails between the DAX and the DJIA returns distributions on short time scales in more detail. Here we only indicate that among the sources of discrepancy between the stock and the index distributions is the existence of periods when the price of a stock does not change. This situation is specific only to very short time scales and results in a number of zero returns in the data. Such returns, of course, influence the average volatility of price changes and thus also affect the normalization, giving broader distributions of the returns while not affecting the tail's slope. For the indices, however, this effect can be neglected even at 1-minute time scale.

The outlying points in the distributions, being especially evident in DJIA for 1 min returns (upper left panel of Fig. 2(b)) but also to a lesser extent in DAX, can be almost exclusively related to large jumps of the price of a single asset which enters the index with a significant weight. Such jumps occur occasionally after some important information reaches the market (financial reports, company fussions, takeovers etc.); the two extreme examples are the $15 \%$ increase and the over 20\% decrease of the IBM share price on Apr 22, 1999 and Oct 21, 1999 openings, respectively. Due to the fact that these single-stock jumps alone caused about 1\% change of the DJIA value, the event which is rather unusual in the time-period analyzed, we decided to remove these IBM stock returns and the two corresponding DJIA returns from our time series for all the time scales. This does not influence the essential results of our analysis in any case.

After calculating $\lambda_{1}\left(w_{j}\right)$ for all $j$ 's, we define and select the windows with strong and weak correlations between the companies. We introduce three distinct kinds of window selection characterized by the following values of the parameters: $\zeta^{W}=0.5$ and $\zeta^{S}=0.5$ (one half of the windows are considered as covering weakly collective trading, and the other half - strongly collective trading), $\zeta^{W}=0.2$ and $\zeta^{S}=0.8$ (20\% of windows with smallest $\lambda_{1}, 20 \%$ of windows with highest $\left.\lambda_{1}\right), \zeta^{W}=0.05$ and $\zeta^{S}=0.95$ (5\% smallest, $5 \%$ highest).

Figure 3 displays the cumulative distributions of the stock returns (dashed) and the index returns (solid) according to the local inter-stock correlation strength ( $W$ - gray, $S$ - black) for the DAX (left panels) and for the DJI (right panels) markets. We present only one time scale $\Delta t=5 \mathrm{~min}$, but these results 


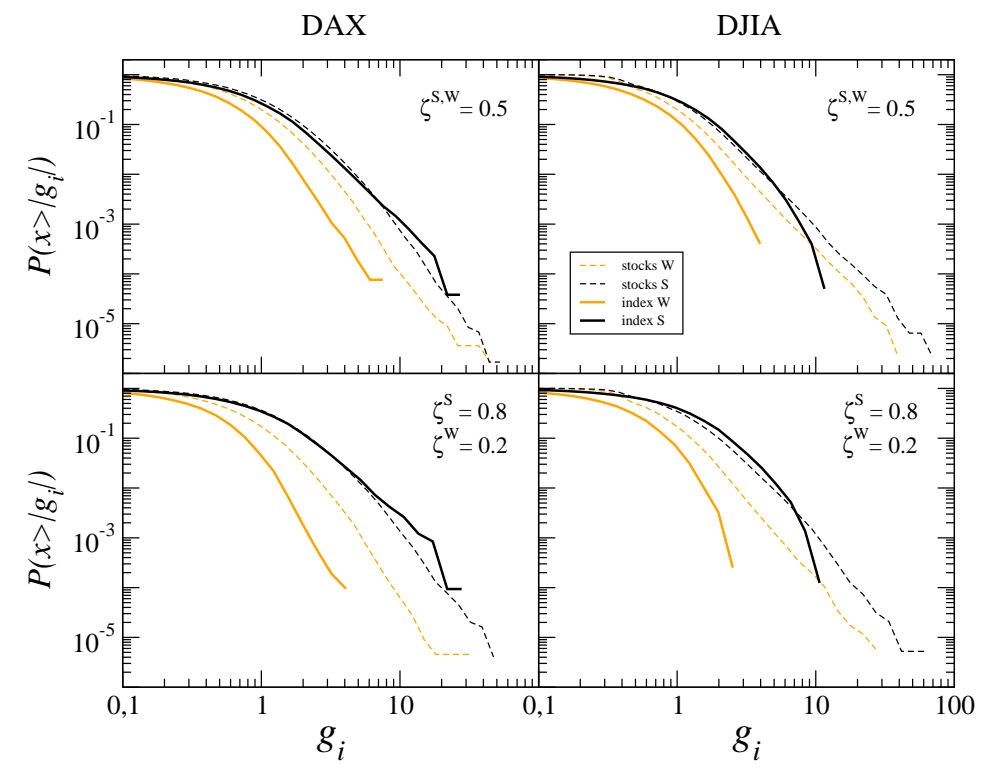

Fig. 3. Cumulative distributions of 5 min stock and index returns for DAX (left column) and DJIA (right column) without the repeated normalization. The distributions of returns corresponding to time windows of weak (W) and strong (S) correlations are presented together in each panel. The upper panels show distributions calculated for the returns from $50 \%$ of the windows with highest value of $\lambda_{1}$ $\left(\zeta^{S}=0.5\right)$ and from the remaining $50 \%$ of the windows with small $\lambda_{1}\left(\zeta^{W}=0.5\right)$. The lower panels display the same for $20 \%$ of the windows with highest $\left(\zeta^{S}=0.8\right)$ and smallest $\left(\zeta^{W}=0.2\right)$ correlations. Gray dashed lines exhibit stock returns distributions for the $\mathrm{W}$ case and black dashed lines for the $\mathrm{S}$ case, while the corresponding index returns distributions are denoted by solid lines: gray (W) and black (S). Note that the two returns corresponding to IBM large jumps on Apr 22, 1999 and Oct 21, 1999 have been removed before calculation of the distributions both for the DJI stocks and for DJIA; the same refers to all subsequent figures.

are qualitatively stable across all analyzed scales. For the companies, both the $W$ and $S$ distributions (which we shall denote as $D A X_{C}^{W}, D A X_{C}^{S}, D J I A_{C}^{W}$ and $D J I A_{C}^{S}$ ) have similar slopes with $D A X_{C}^{W}$ and $D J I A_{C}^{W}$ slightly shifted to the left compared to $D A X_{C}^{S}$ and $D J I A_{C}^{S}$. This shift is caused by different variance of the distribution in the $W$ and $S$ cases and it illustrates the already known fact, that large price fluctuations (volatility) are more likely to happen when the market is more collective (and vice versa) $[14,15]$. In fact, the difference between the distributions enlarges with increasing $\Delta \zeta:=\zeta^{S}-\zeta^{W}$.

Another interesting conclusion can be drawn from the distributions of index returns (these we denote as $D A X_{I}^{W}, D A X_{I}^{S}, D J I A_{I}^{W}$ and $D J I A_{I}^{S}$ ). Here the differences between the $W$ and $S$ distributions, for both of the indices, are amplified compared to the stock returns, which is evident both for $\zeta^{W, S}=0.5$ and $\zeta^{W}=0.2, \zeta^{S}=0.8$, with the separation much larger in the latter case. As such a large difference cannot be explained merely on the basis of the 
divergence between the associated distributions for the companies, the range of correlations may be the crucial factor here.

Although some conclusion on the shape of the distributions in their tails can be drawn directly from the lower panels of Fig. 3, a real comparison may be performed only if all the sets of the returns are normalized once again to have a unit variance (so far the variance is different within each of the interrelated $W$ and $S$ sets). Figure 4 is the central point of the present paper. It exhibits the cumulative distributions of the stock and index returns after the repeated normalization. Parts (a)-(d) show the results for $\Delta t=1,5,10$ and $30 \mathrm{~min}$, respectively.

The main conclusions which can be drawn from Figure 4 are as follows:

(i) The cumulative distributions of the stock returns (dashed lines) for $W$ and $S$ windows (gray and black, respectively) reveal roughly similar properties both in their central parts and the tails, regardless of the market, the time scale $\Delta t$ and the threshold separation $\Delta \zeta$. A perfect agreement of the distributions is evident for the DJI stocks, while some small differences can be observed for the German stocks, especially for short time scales.

(ii) On the contrary, the distributions of the index fluctuations (solid lines) are heavily influenced by the strength of correlations between the stock price movements. The distributions of the returns in $W$ windows (grey solid line) tend to be more Gaussian than the ones associated with $S$ windows (black solid line). This systematically applies to almost all cases with an exception for $D J I A_{I}^{W}$ and $D J I A_{I}^{S}$ computed for $\Delta t=1 \mathrm{~min}$, where the situation is less clear. Even in this case, however, the two distributions seem to differ for the largest $\Delta \zeta$ (bottom right panel of Fig. $4(\mathrm{a})$ ). For $\Delta t=5 \mathrm{~min}$ and $\Delta \zeta=0.9$ we do not observe so big difference between the distributions in DJIA as we might expect, but this fact can well be attributed to poor statistics (in this case, each of the two distributions was calculated from 2100 returns only).

(iii) The larger $\Delta \zeta$ is, the larger is the difference between $D A X_{I}^{S}$ and $D A X_{I}^{W}$; this rule is especially significant at small $\Delta t$ and deminishes at larger $\Delta t$. For DJIA, the analogous increase of separation is less pronounced (Fig. 4(a)-4(d)).

(iv) The above-mentioned increase of separation between $D A X_{I}^{S}$ and $D A X_{I}^{W}$ with increasing $\Delta \zeta$ is associated with the appearence of the distribution with scaling regions whose slope is in the Lévy-stable regime. Yet another look at Fig. 2(a) allows one to see that even though the distributions of the DAX returns show fat tails, they are by no means stable. A significantly different behaviour of the distribution can be obtained after filtering out those returns that belong to the intervals of uncorrelated trading and whose distribution drops down considerably faster (Fig. 4(a)). With the threshold as low as at $\zeta^{S}=0.5$ (i.e. $\Lambda^{S}=5.76$ ), the $D A X_{I}^{S}$ distribution presents the apparent scaling 

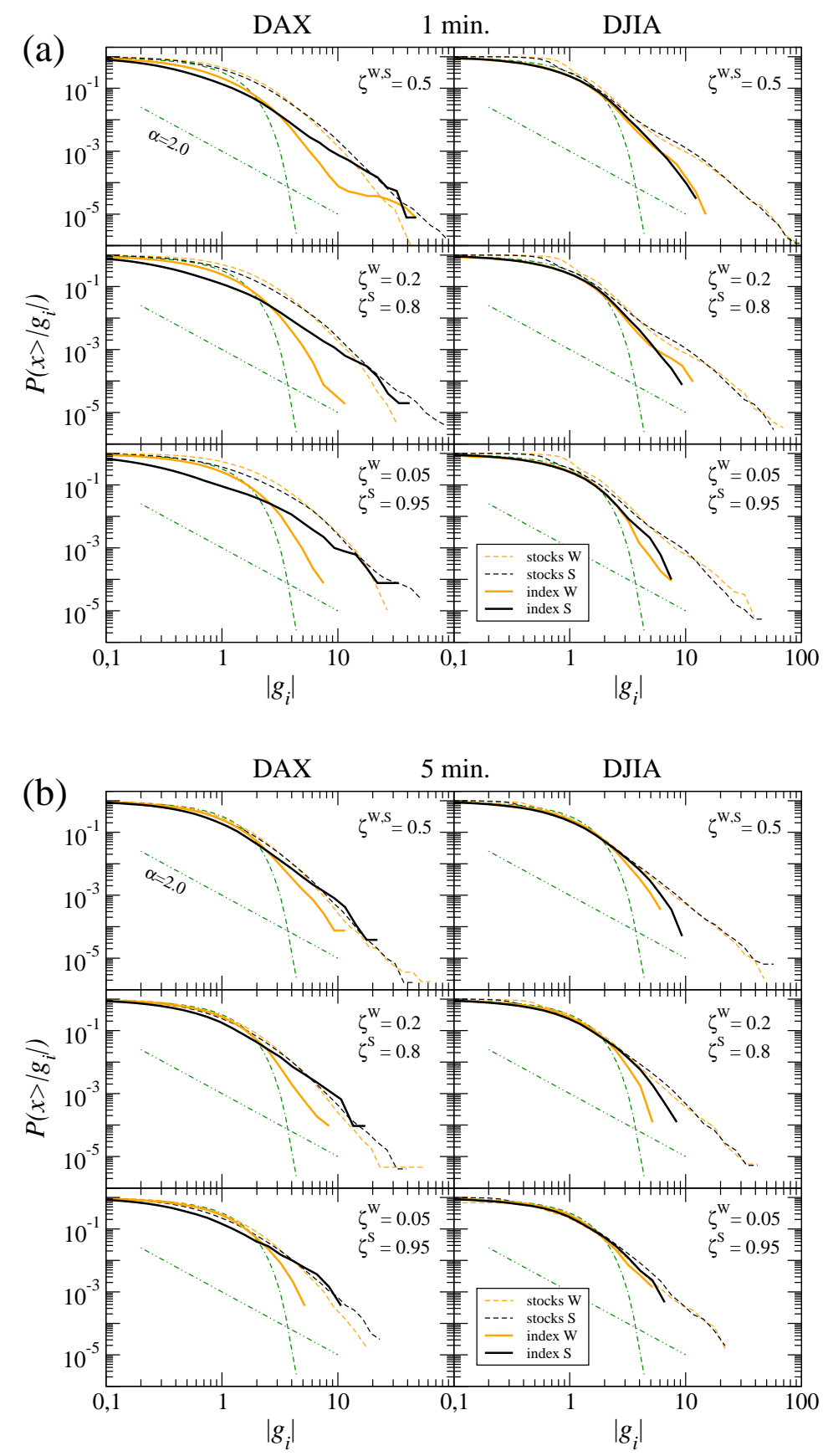

Fig. 4. Cumulative distributions of stock and index returns after the repeated normalization (see text) for the DAX (left) and the DJIA (right) markets and for four different time scales: $1 \mathrm{~min}$ (a), $5 \mathrm{~min}$ (b), $10 \mathrm{~min}$ (c) and $30 \mathrm{~min}$ (d). The distributions are denoted as in Fig. 3. In each panel, the cumulative normal distribution is denoted by a dash-dotted line while the Lévy stable regime $(\alpha=2.0)$ is denoted by a dash-double-dotted slanted line. In (c) and (d) no extreme distributions $\left(\zeta^{W}=0.05\right.$ and $\left.\zeta^{S}=0.95\right)$ can be presented due to too poor statistics. 

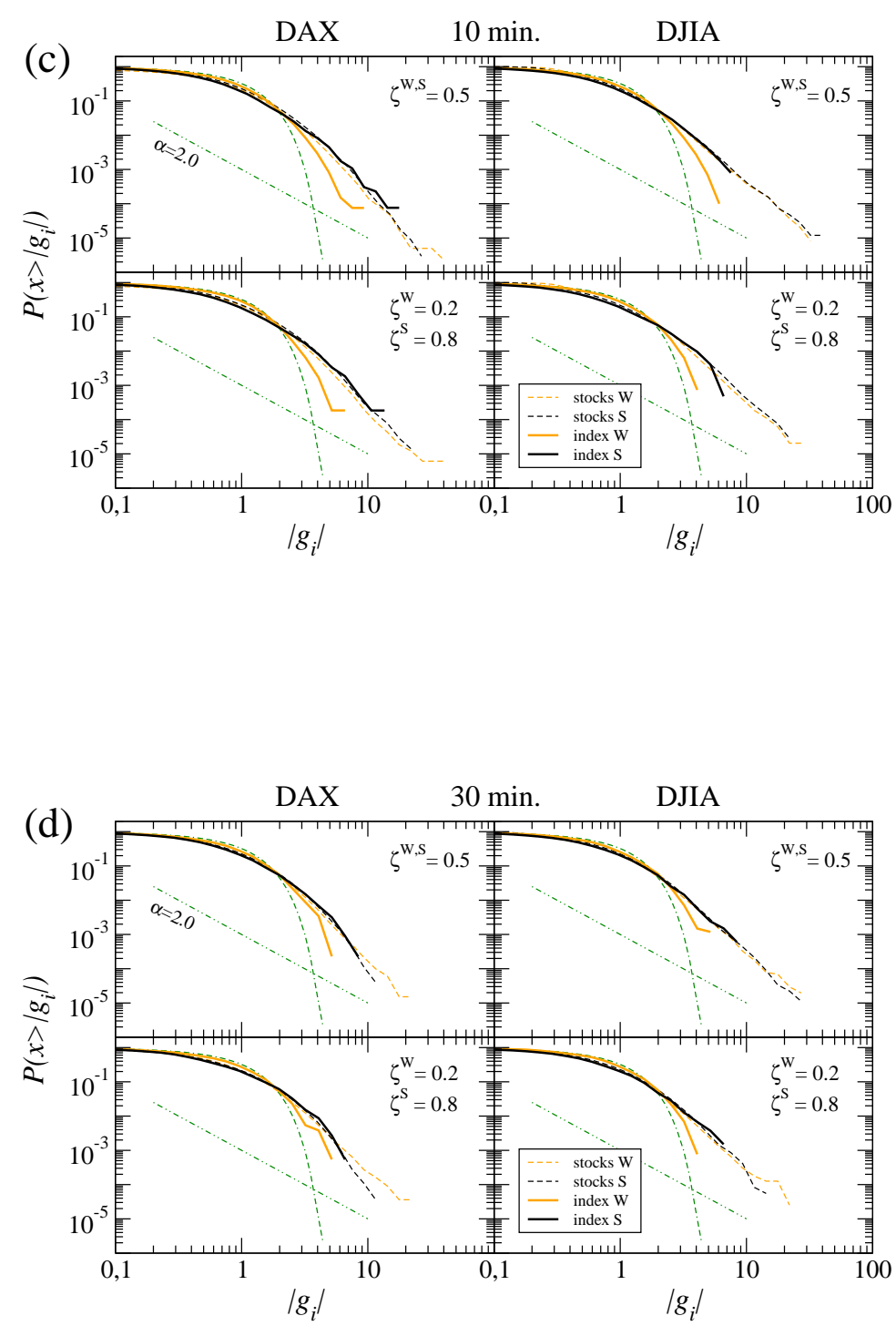

Fig. 4. Continued.

just at the edge of the Lévy-stable regime $(\alpha \simeq 2.0$, represented by the slant dash-dotted line in each panel). By rising the threshold $\zeta^{S} \geq 0.8\left(\Lambda^{S} \geq 7.90\right)$ we observe the occurence and inflation of another scaling region for smaller values of the returns with the scaling index deep inside the stable range $(\alpha \simeq$ 1.3). For $\Delta t=5 \mathrm{~min}$ (Fig. $4(\mathrm{~b})$ ) we also identify the scaling region but now with $\alpha$ continuously decreasing with increasing $\zeta^{S}$. This region is shorter than the one for $\Delta t=1 \mathrm{~min}$ and falls into the stable regime at significantly higher threshold $\left(\zeta^{S} \simeq 0.8\right)$. No scaling of $D A X_{I}^{S}$ can be observed for larger time scales (Fig. 4(c) and (d)). Unlike DAX, DJIA does not convincingly scale for 
any of the analyzed values of $\Delta t$ (compare ref. [5]).

A striking difference between the properties of the DJIA and the DAX returns distributions can be seen for the time scale of 1 min (Fig. 4(a)). For DAX, the distributions for the $S$ and $W$ windows are disparate, whereas for DJIA they have similar shape. One of the possible sources of this discrepancy can be the already-mentioned more noisy evolution of the DJI market. Indeed, our computation shows that if $\Delta t=1 \mathrm{~min}$, the median of $P\left(\lambda_{1}\left(w_{j}\right)\right)$ reaches only 4.22 for the DJI stocks; this value does not stray much from the upper edge of the eigenvalue spectrum of a Wishart matrix. Thus, even the relatively high values of $\lambda_{1}\left(w_{j}\right)$ are not very distant from the random case, e.g. for the middle right panel of Fig. 4(a), $\zeta^{S}=0.8$ corresponds to the threshold $\Lambda^{S}=5.08$. This can well account for the fact documented in the bottom right panel of Fig. 4(a) that the $D J I A_{I}^{S}$ and the $D J I A_{I}^{W}$ distributions start to significantly differ only for $\zeta^{S}=0.95\left(\Lambda^{S}=6.40\right)$. For comparison, the threshold values $\Lambda^{S}$ for DAX stocks are as follows: $5.76\left(\zeta^{S}=0.5\right), 7.90\left(\zeta^{S}=0.8\right)$ and $10.60\left(\zeta^{S}=0.95\right)$. The strongest correlations and large values of $\lambda_{1}\left(w_{j}\right)$ are more likely to occur at market-specific periods of intraday trading [23,12,24,13], especially in the German market; such periods are usually associated with highly volatile behaviour of the market and rapid collective movements of prices. An example can be the sudden significant changes of DAX which frequently occur almost precisely at 14:30 [12,13]. These changes are visible predominantly on short time scales; on longer time scales they are often averaged out and vanish. This can be considered as one of the possible sources of larger collectivity of DAX on short time scales. Another source is the strong influence of the NASDAQ and NYSE evolution on the Frankfurt stock market as a whole. Daily pattern of $\lambda_{1}\left(w_{j}\right)$ fluctuations reveals significant increase of the largest eigenvalue's magnitude after 15:30 (i.e. 9:30 in New York) when trading starts on the NYSE and NASDAQ markets; also the 14:30 peak is caused by certain external factors. From this point of view the American market can be considered as being relatively independent and, thus, less correlated. However, as the big difference between $D A X_{I}^{W}$ and $D A X_{I}^{S}$ (and between the distributions of the DAX and the DJIA returns) cannot be attributed merely to the strongly correlated dynamics of the German market, an explanation for the observed shape of the distributions of the DAX returns requires more extensive investigation.

A potential source of the fat-tailed distributions of the DAX returns may be the calculation procedure of DAX after the market opening: an opening value may be assigned to the index only after not less than $50 \%$ of the DAX component stocks representing at least $70 \%$ of the total DAX market capitalization have already been traded on a given day. This usually happens a few minutes after the actual market opening and leads to a significant relative amplifiction of the overnight DAX return if the corresponding time scale is shorter than a few minutes (as it is in our case of $\Delta t=1 \mathrm{~min}$ ). Obviously, for any longer time scale this effect should not influence the magnitude of the corresponding 

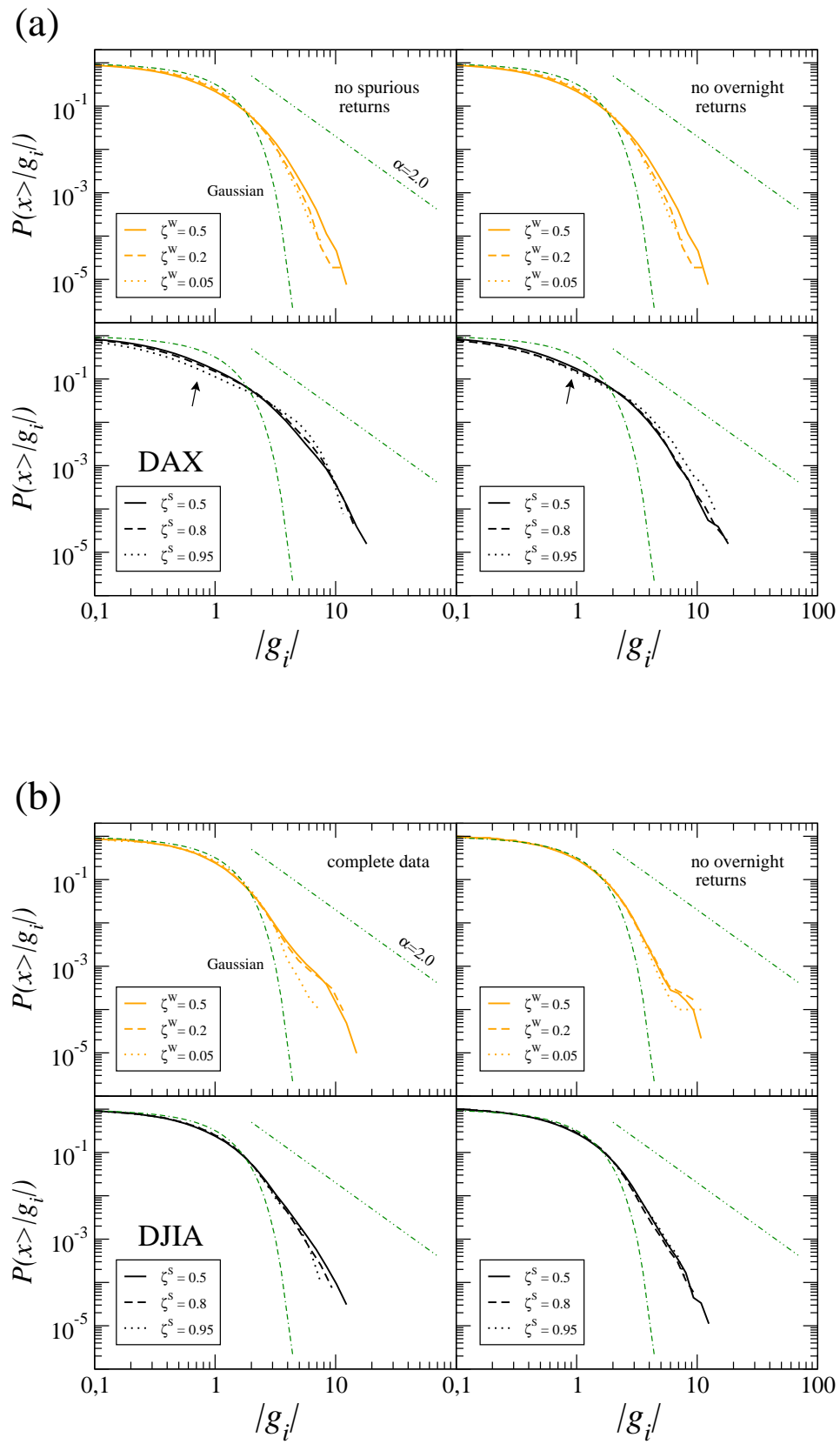

Fig. 5. Cumulative distributions of 1 min returns for DAX (a) and DJIA (b). Three different realizations of the $W$ case (upper panels) and the $S$ case (lower panels) are presented. For DAX, panels on the left side of (a) present distributions calculated from data without spurious overnight returns, while panels on the right side present the same distributions after removing all the overnight returns. Left-hand side of (b) corresponds to complete DJIA data (i.e. the same as in Fig. 4(a)) and the right-hand side shows the same distributions but now comprising no overnight returns. The cumulative Gaussian and Lévy stable distributions are also shown (dash-dotted lines). The small arrows in (a) point to the Lévy scaling regions. 
returns; in fact, Figs. 2(a), 4(c) and 4(d) show that the $S$ distributions for DJIA and DAX look similar both for $\Delta t=10$ and for $\Delta t=30 \mathrm{~min}$. In order to quantify the influence of the overnight DAX returns on the corresponding distributions, we take our time series of 1 minute returns of DAX and select all the returns which correspond to overnight changes of the index value (i.e. the difference between a previous day's closing value and the next day's opening value). Next we zero the spurious returns corresponding to the situation in which the opening value was assigned to DAX later than at 8:31:00 (as they should not be considered as valid 1 min returns), and leave the remaining overnight returns unchanged (roughly about half the total number of overnight returns). Then we calculate the distributions according to the same procedure as for the complete time series in Fig. 4(a). For a comparison, we also calculate the distributions after zeroing all the overnight returns both the spurious and the valid ones.

Figure 5(a) exhibits the cumulative distributions of the DAX returns taken from the $W$ windows (top panels) and the $S$ windows (bottom panels) separately; in each panel distributions for three different values of $\zeta^{W}$ or $\zeta^{S}$ are shown. Both the distributions calculated from the data without the spurious returns (on the left of Figure) and the distributions calculated from the data without all the overnight returns (on the right) have got significantly thinner tails than their counterparts for complete data in Fig. 4(a); this is true both for the collective and for the uncorrelated trading intervals. Moreover, the outer scaling region with $\alpha \simeq 2.0$ which was clearly visible in Fig. 4(a), here disappeared, which permits us to identify its origin as being merely due to the calculation procedure of the DAX opening value. The extremely fat tails for DAX observed in Fig. 4(a) may therefore be considered as an external effect unrelated to the inner properties of the market dynamics. These fat tails and scaling are more significant for the $S$ windows than for the $W$ ones, but this is purely accidental: the opening 30 minutes covered by the first window on each day is usually associated with collective dynamics of stocks and thus it is classified as an $S$ window.

In contrast to the outer scaling region with $\alpha=2.0$, the inner scaling region characterized by $\alpha=1.3$ and which is best visible for high values of $\zeta^{S}$ for $0.5<\left|g_{i}\right|<3$ (arrows in Figure), survives. Removing the spurious returns does not affect it at all and removing also the rest of overnight returns only slightly shorten it and change its slope to 1.4. A similar region of scaling in the Lévy-stable regime has already been identified previously for the S\&P 500 index [5]; in that case $\alpha=1.6$. Interestingly, a trace of a related feature (but without clear scaling) can also be seen for the DAX-stocks returns in Fig. 4(a) (bottom left panel, black dashed line). As this stable scaling region occurs only in "collective" windows with large $\lambda_{1}\left(w_{j}\right)$ it seems that, for DAX, its origin cannot be attributed to the quantization of the returns as authors of ref. [5] hypothesize for S\&P500. It is also interesting to note that after 


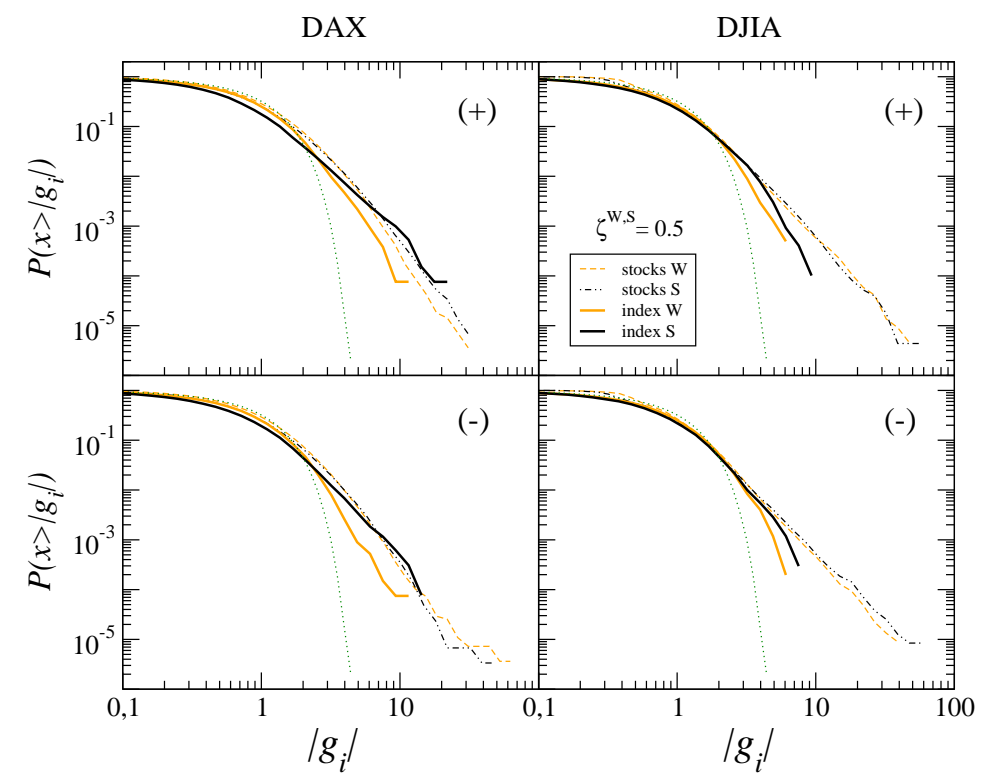

Fig. 6. Cumulative distributions of 5 min stock and index returns (complete data and repeated normalization) for the DAX (left) and the DJIA (right) markets. The upper (lower) panels correspond to positive (negative) fluctuations. Only the case of $\zeta^{W, S}=0.5$ is shown. The cumulative normal distribution is denoted by a dotted line in each panel.

removing the spurious returns, the distributions for the $S$-windows closely resemble the distributions for the truncated Lévy processes, where the central part is a Lévy distribution while, owing to the exponential cut-off of tails, the second moment is finite (see ref. [2]). In fact, if we calculate pdf of the price increments (instead of the log-returns) for the $S$-windows, we obtain good fits by using Lévy distributions with $\alpha=1.3 \div 1.4$; the tails of such pdf's decay approx. exponentially with the best agreement being observed for $\zeta^{S}=0.8$. After removing all the overnight returns, the tails do not present explicit exponential decay but second moment is obviously still finite (bottom right panel of Fig. 5(a)).

Figure 5(b) displays the results of an analogous analysis for DJIA. Due to the fact that the opening values of DJIA may be calculated without any restriction, there is no spurious returns and thus in Figure we show the results for the complete data instead: the distributions on the left side are exactly the same as those on the right side of Fig. 4(a). Even if we remove all the overnight returns we do not observe any qualitative modification of the distribution's shape (right side of Fig. 5(b)). Only small quantitative changes can be seen here; without the opening returns, the $D J I A_{I}^{W}$ distributions are characterised by slightly thinner tails and start to differ from their $S$ counterparts already for $\zeta^{S}=0.8$ instead of $\zeta^{S}=0.95$ as it was for the complete data. This is not shown explicitely but can be inferred from a careful inspection of the 
right-side panels of Fig. 5(b). For DAX (Fig. 5(a)), the difference between the distributions corresponding to $W$ and $S$ windows decreases both after removing the spurious and the valid overnight returns, but it is still stronger for DAX than for DJIA and increases with increasing $\Delta \zeta$.

For longer time scales $(\Delta t \geq 5 \mathrm{~min})$, removing the spurious overnight returns does not influence the results presented in Fig. 4(b)-(d) for any of the two markets whereas removing also the valid overnight returns influence the distributions. For $\Delta t \geq 10 \mathrm{~min}$, the overnight returns share the properties with intraday returns and removing them has no qualitative effect. Thus, we may conclude that the large difference in the distributions of the returns between DAX and DJIA observed in Fig. 4(a) is predominantly due to the following two factors: (a) the properties of the overnight returns and especially the nontrivial calculation of such returns in DAX, and (b) the different strength of couplings in each of the two markets; the DJI stocks present more noisy evolution than the German stocks, which leads to similarity of the correlation properties of the $W$ and $S$ intervals and, in turn, similarity of the corresponding distributions in DJIA.

Finally, we shall compare the properties of the distributions of the positive and negative returns $[5,25]$. Such distributions for $\Delta t=5 \mathrm{~min}$ are displayed in Figure 6 both for the German (left) and the American (right) markets. The positive fluctuations corresponding to index drawups (upper panels) and the negative ones associated with index drawdowns (lower panels) do not differ from each other qualitatively, resembling the distributions for the absolute returns presented for this time scale in Fig. 4(b). This is in agreement with the findings of ref. [4,5] that the properties of distributions of the stock and index returns are symmetric with respect to zero.

\section{Conclusions}

To summarize, the results of our analysis show that the time intervals characterized by strongly collective behaviour of stocks are associated with the distributions of the index returns, whose properties differ from the ones for the intervals dominated by noise. Strongly correlated market can be related to the phenomenon of fat tails of the returns distribution, while faster convergence of such a distribution to normal can be attributed to a decorrelated trading. This might be considered as an empirical argument supporting the hypothesis stating that the important factor responsible for the fat tails of the distributions of the index returns is the inter-stock correlations [5]. Such an effect is observed in both the German and the American market for time scales of at least $5 \mathrm{~min}$ and in the German market for even 1 min time scale. This does not exclude, however, possible influence of other factors which can 
either amplify the effect of inter-stock correlations or be a distinct source of the non-Gaussian tails (see [25]). For the DAX market, which is in principle more collective than the Dow Jones one on short time scales $[10,26]$, the strong inter-stock couplings which occur both repeatedly in specific periods of a trading day and uniquely at random moments, lead to the occurence of the Lévy-stable region in the distributions of the index returns. This region, however, comprises returns of moderate size only and its existence does not affect the distributions' tails. The results of our study indicate that removing spurious overnight returns in the $S$-windows leads to the distributions which, on short time scales, closely resemble those for the truncated Lévy processes. The existence of and switching between different fluctuation regimes in index evolution during periods of correlated and decorrelated trading resembles the phenomenon of two-phase behaviour of the demand for stocks where the equilibrium and the out-of-equilibrium phase interweave [27]. We do not observe, however, any sudden change of properties of the fluctuations for any of the values of the control parameter $\lambda_{1}$, but rather a continuous transition from one type of behaviour to another type, which is best visible for $1 \mathrm{~min}$ and 5 min returns of DAX.

\section{References}

[1] B. Mandelbrot, J. Business 36 (1963) 394-419

[2] R.N. Mantegna, H.E. Stanley, Nature 376 (1995) 46-49

[3] T. Lux, Appl. Fin. Econ. 6 (1996) 463- 475

[4] V. Plerou, P. Gopikrishnan, L.A.N. Amaral, M. Meyer, H.E. Stanley, Phys. Rev. E 60 (1999) 6519-6529

[5] P. Gopikrishnan, V. Plerou, L.A.N. Amaral, M. Meyer, H.E. Stanley, Phys. Rev. E 60 (1999) 5305-5316

[6] S. Drożdż, J. Kwapień, F. Grümmer, F. Ruf, J. Speth, Are the contemporary financial fluctuations sooner converging to normal?, cond-mat/0208240 (2002)

[7] M.L. Mehta Random Matrices, Academic Press (Boston 1991)

[8] L. Laloux, P. Cizeau, J.-P. Bouchaud, M. Potters, Phys. Rev. Lett. 83 (1999) $1467-1470$

[9] V. Plerou, P. Gopikrishnan, B. Rosenow, L.A.N. Amaral, H.E. Stanley, Phys. Rev. Lett. 83 (1999) 1471-1474

[10] S. Drożdż, F. Grümmer, A.Z. Górski, F. Ruf, J. Speth, Physica A 287 (2000) 440-449

[11] S. Drożdż, F. Grümmer, F. Ruf, J. Speth, Physica A 294 (2001) 226 
[12] S. Drożdż, J. Kwapień, F. Grümmer, F. Ruf, J. Speth, Physica A 299 (2001) 144-153

[13] J. Kwapień, S. Drożdż, F. Grümmer, F. Ruf, J. Speth, Physica A 309 (2002) $171-182$

[14] P. Cizeau, M. Potters, J.-P. Bouchaud, Quant. Fin. 1 (2001) 217-222

[15] C. Mounfield, P. Ormerod, Market Correlation and Market Volatility in US Blue Chip Stocks, Crowell Memorial Prize Submission (2001)

[16] http://www.taq.com (data from NYSE) and H. Goeppl, Karlsruher Kapitalmarktdatenbank (KKMDB), Institut für Entscheidungstheorie u. Unternehmensforschung, Universität Karlsruhe (TH) (data from Deutsche Börse)

[17] A.M. Sengupta, P.P. Mitra, Phys. Rev. E 60 (1999) 3389-3392

[18] P. Gopikrishnan, B. Rosenow, V. Plerou, H.E. Stanley, Phys. Rev. E 64 (2001) 035106(R)-1(74)

[19] V. Plerou, P. Gopikrishnan, B. Rosenow, L.A.N. Amaral, T. Guhr, H.E. Stanley, Phys. Rev. E 65 (2002) 066126

[20] J.-P. Onnela, A. Chakraborti, K. Kaski, K. Kertész, A. Kanto, Dynamics of market correlations: Taxonomy and portfolio analysis, cond-mat/0302546 (2003)

[21] C.A. Tracy, H. Widom, The Distribution of the Largest Eigenvalue in the Gaussian Ensembles: $\beta=1,2,4$, solv-int/9707001 (1997)

[22] I.M. Johnstone, Ann. Stat. 29 (2001) 295

[23] Y. Liu, P. Gopikrishnan, P. Cizeau, M. Meyer, C.-K. Peng, H.E. Stanley, Phys. Rev. E 60 (1999) 1390-1400

[24] B.H. Wang, P.M. Hui, Eur. Phys. J. B 20 (2001) 573-579

[25] F. Lillo, R.N. Mantegna, Eur. Phys. J. B 15 (2000) 603-606;

F. Lillo, R.N. Mantegna, Phys. Rev. E 62 (2000) 6126-6134;

F. Lillo, R.N. Mantegna, Physica A 299 (2001) 161-167

[26] S. Drożdż, F. Grümmer, F. Ruf, J. Speth, Empirical Science of Financial Fluctuations, H. Takayasu (Ed.), (Springer-Verlag Tokio 2002) 41

[27] V. Plerou, P. Gopikrishnan, H.E. Stanley, Nature 421 (2003) 130;

M. Potters, J.-P. Bouchaud, Comment on "Two-phase behavior of financial markets", cond-mat/0304451 (2003) 